\title{
Influence of the prosthetic restoration design on the peri-implant bone loss.
}

Georgina García-Engra; Ana Roig-Vanaclocha; Ruben Agustín-Panadero; Lucía Fernández-Estevan; María Fernanda Solá Ruiz.

Master of Prosthodontic and Occlusion.Department of Stomatology. University of Valencia.

\section{Abstract}

Background: The rehabilitation of missing teeth through dental implants is a conservative approach. There are many factor that affects the success of implants among which we find the design of the implant-supported restoration.

Aim: The aim of this study was to analyse the peri-implant bone loss a year after prosthetic loading, assessing the influence of the design of the prosthetic restoration.

Methods and Materials: A prospective study of 62 dental implants was carried out distributed in 62 dental implants was carried out distributed in three groups according to their non-cantilevered
restoration. Peri-implant Bone loss was assessed from a periapical radiograph using the AutoCAD® software.

Results: After one year from loading, the bone loss values were $0,95 \mathrm{~mm}$. The statistical analysis showed that the type of prosthodontic restoration placed and the location of the implant did not have statistically significan differences in the bone loss.

Conclusions: The mean values of bone loss found in this study are within the range considered acceptable in the literature Statistically significant differences were not Staund between the type of prosthetic restoration found between the type of prosthetic restoration and the location of the implant. Therefore, more
relevant studies would be needed to obtain a relevant studies would be

\section{Background and Aim}

Oral rehabilitation with dental implants is a consolidated procedure for restoring missing teeth, which has proven long-term success.

One of the criteria for determining the success of an implant is the marginal bone loss. A bone loss of $1,5 \mathrm{~mm}$ during the first year is within the range considered acceptable in the literature.

There are many factors that affect bone loss around implants. It can be influenced and aggravated by a finite number of elements such as occlusal stress, surgical trauma, implantrelated properties, implant-crown ratio, prosthetic characteristics and patient-specific risk factors. From a biomechanical point of view we find the design of the implant-supported restoration.

Since dental implants are increasingly being placed and multiple factors can cause bone loss around them, we believe it is important to consider if the prosthodontic design affects the peri-implant bone loss.

\section{THE AIM of this study was:}

1. To analyze the peri-implant bone loss a year after prosthetic loading.

2. Assessing the influence of the design of the prosthetic restoration between implant.

3. To Evaluate the influence of the position and localitation of implants in the peri-implant bone loss.

\section{Methods and Materials}

- A prospective study of 62 dental implants was carried out. The implants were placed in 23 patients between May 2013 and May 2014 at the Unit of Prosthodontics of the 2014 at the Unit of
University of Valencia.

Kohno implants were used from Sweden\&Martina ${ }^{\circledR} \quad$ (Sweden\&Martina ${ }^{\circledR}$ SPA. Padova, Italia) and they were distributed in three groups according to their noncantilevered restoration: two splinted crowns(Group 1) , three-unit and four-unit fixed partial restorations supported by two dental implants (group 2 and 3 ).

SAMPLE $(n=62)$

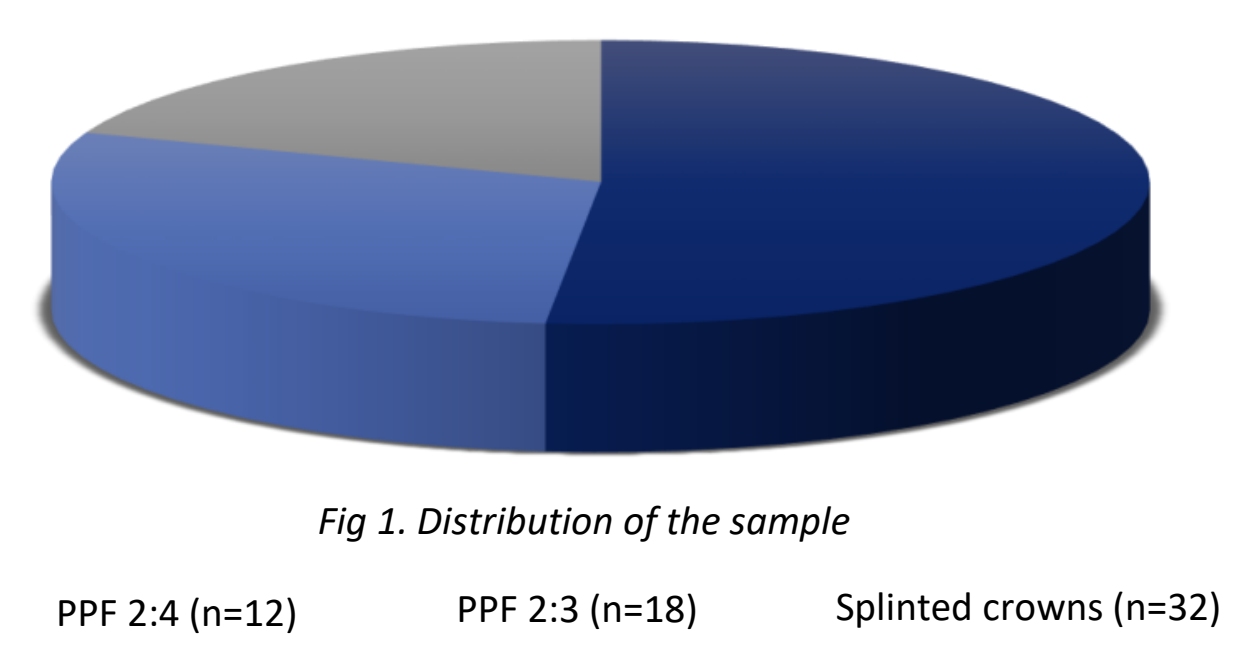

- Bone loss, three years after loading, was assessed from a periapical radiograph using the AutoCAD® software (Autodesk Inc., the AutoCADß
California, EE.UU.).

Two reference points were localized at the platform of the implant in mesial and distal side. A straight line was drawn between them creating the base height axis. (Figure 2).

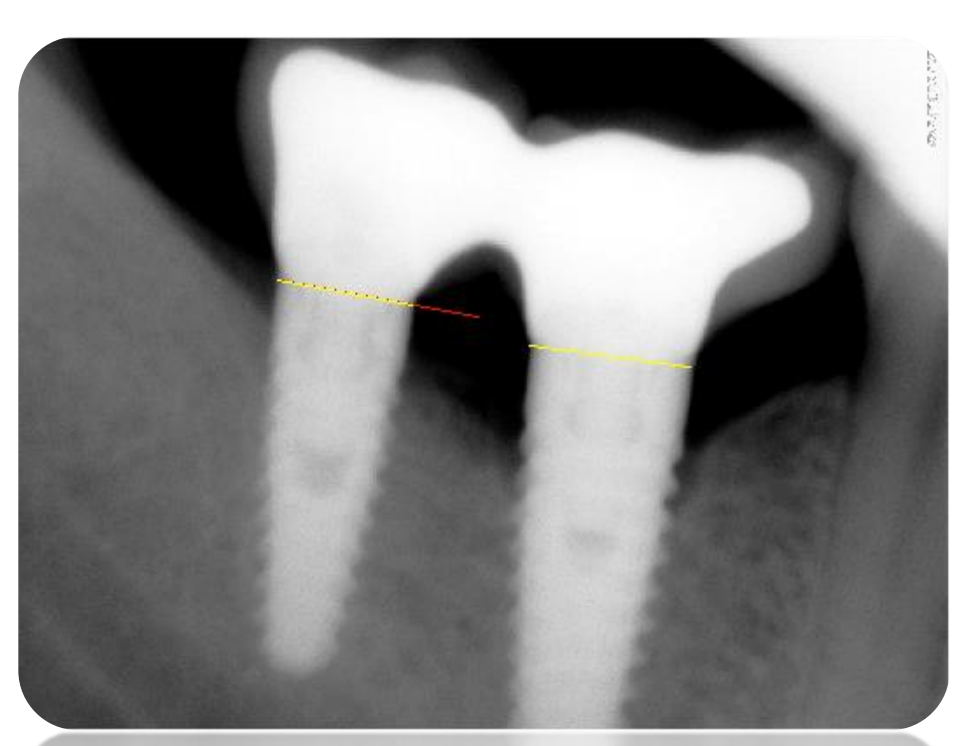

Fig 2. Straigh line and base height axis.

- A perpendicular line was drawn in each side to the base height axis from bone leve (figure 3). The height of the polished implant neck was rested $(0,8 \mathrm{~mm})$ to each mesurement.

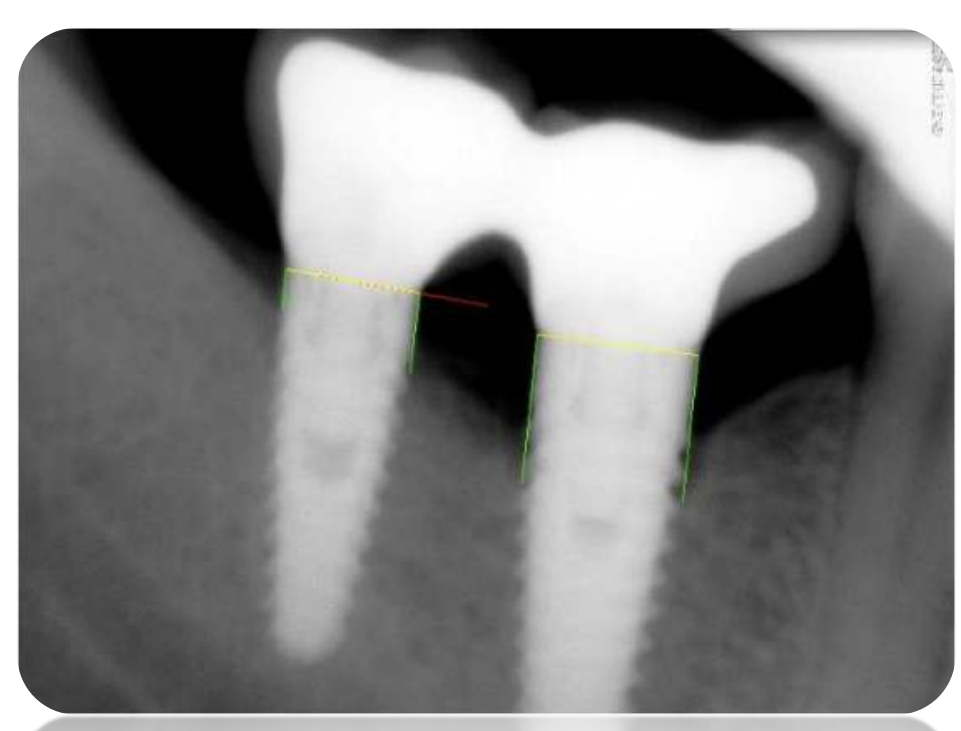

Fig 3. Mesial and distal bone loss.

- The differences between the baseline radiograph and after one year of load radiograph let us to calculate the mean in mesial and distal sides. This was the reference value of peri-implant bone lost.

\section{Results}

The global peri-implant bone loss values obtained after one year from loading was $0.95 \mathrm{~mm}$. This result is considered within the acceptable values.

The peri-implant bone loss was evaluated according to the design of the restoration used, to the position of the implant within the prosthesis and to the influence of the position of the implant in the arches.

After the statistical analysis, the type of prosthodontic restoration placed and the location of the implant did not show statistically significant differences in the bone loss.

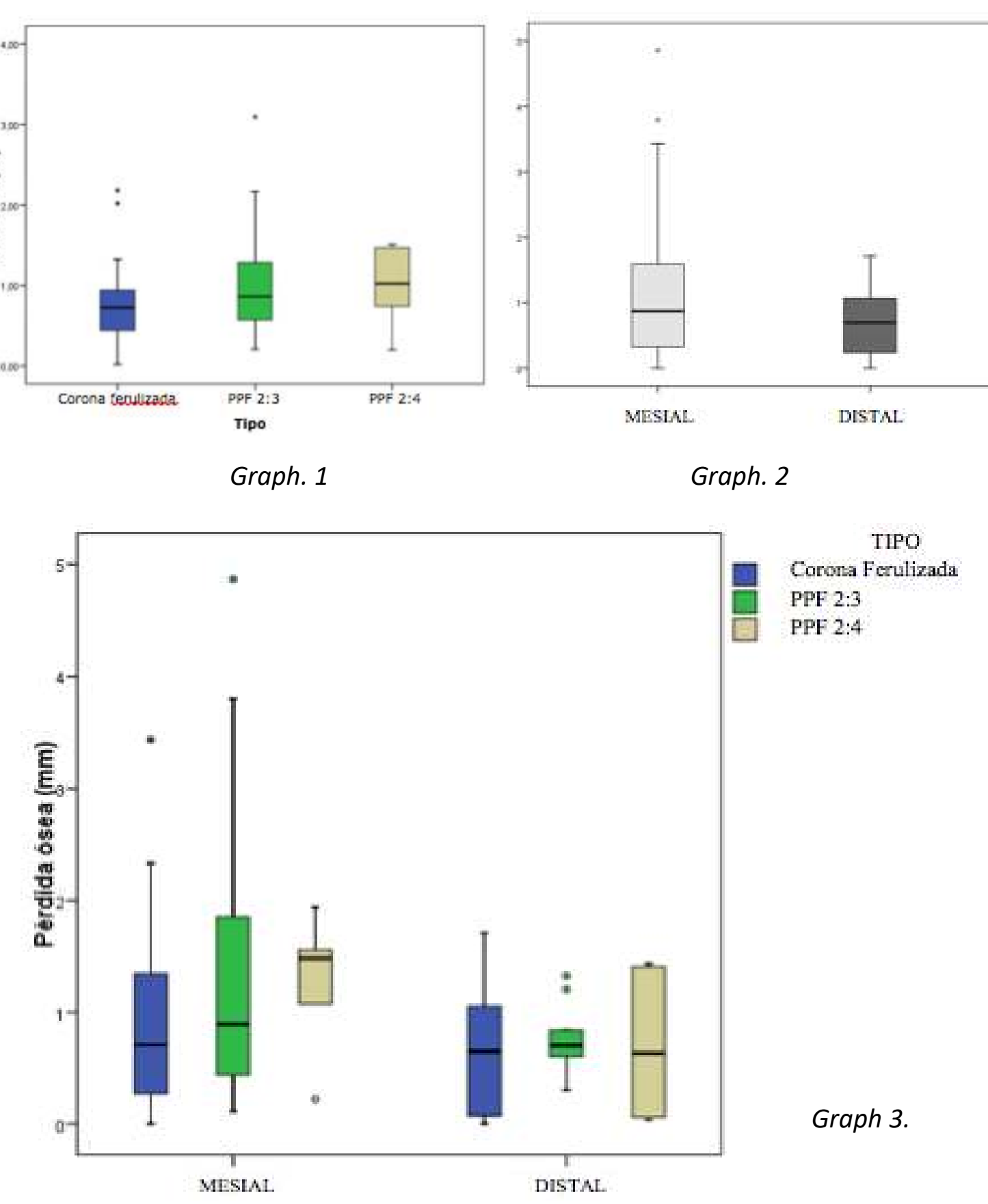

Regarding the type of prosthetic restoration, the higher values were found in three-unit fixed partial restoration supported by two Implants $(1.16 \mathrm{~mm})$, follow by four-unit fixed partia restoration supported by two implants (1.00 $\mathrm{mm})$. However, the two splinted crowns group showed the lowest values of peri-implant bone los $(0.81 \mathrm{~mm})$. On the other hand, implants located in mesial and in the lower arch obtained higher values of bone loss. The mean values for mesial implants was $1.18 \mathrm{~mm}$ while dista implants obtained $0.71 \mathrm{~mm}$ of peri-implant bone loss. In addition, implants located in the lower arch had a mean value of bone loss of $1.06 \mathrm{~mm}$ compared to those located in the upper arch which presented a loss of $0.72 \mathrm{~mm}$.

\section{Conclusions}

Despite the limits of the present study, it can be concluded that mean values of bone loss $(0.95$ $\mathrm{mm}$ after a year of loading) are within the range considered acceptable in the literature.

Statistically significant differences were not found between the type of prosthetic restoration and the location of the implant but the less values of bone loss where found in the Group 1 with a mean of $0,81 \mathrm{~mm}$

Therefore, more relevant studies would be needed to obtain a better scientific evidence.

\section{References}

